\title{
As diferentes tipologias que descrevem o interesse dos jovens brasileiros pelas ciências
}

The different typologies describing the interest of Brazilian youth in Science

Daniel Morin Ocampo ${ }^{1}$

Luiz Caldeira Brant de Tolentino Neto

\begin{abstract}
Resumo
Conhecer os interesses dos estudantes é parte fundamental para facilitar o processo de ensino aprendizagem. Nesse sentido, surgem as investigações que se propõem a compreender como os jovens pensam e se posicionam frente às ciências e tecnologia. Uma destas iniciativas é o projeto Barômetro Brasil, um questionário composto por 96 questões, na escala de Likert de 4 pontos, que investiga, entre outras coisas, o interesse dos jovens frente às ciências e tecnologia. No presente estudo, utilizamos os dados produzidos com a aplicação do Barômetro Brasil, no ano de 2014, oportunidade onde esse instrumento foi aplicado a 2404 estudantes, na faixa dos 15 anos, sendo essa uma amostra representativa da população dos jovens brasileiros, nessa faixa etária. Utilizando o método k-means, para análise de clusters, encontramos 4 tipologias para os estudantes brasileiros: Relutantes, Entusiastas, Indecisos Seletivos, Indecisos Não Seletivos. Além disso, observamos o interesse de cada tipologia em relação a seis temas distintos: Saúde; Mistérios; Inovações Científicas; Agricultura; Ciência e Cientistas; Tecnologia. Esses temas foram determinados utilizando a análise de clusters pelo método hierárquico. A partir deste estudo, concluímos que os temas Mistérios e Inovações Científicas são os que apresentam maior potencial para fomentar a motivação intrínseca dos estudantes, uma vez que são os temas que manifestam interesse acima da média, em todas as quatro tipologias.
\end{abstract}

Palavras chave: Interesse em ciências, Motivação Intrínseca; Tipologia de Estudantes, Barômetro Brasil.

\section{Abstract}

It is essential understanding students' interests to help improving the teaching-learning process. Therefore, investigations aimed at understanding how young people think and feel about Science and Technology have emerged. One of these initiatives lies on the project called Barômetro Brasil, which is a 4-point Likert scale questionnaire comprising 96 questions that investigate the interest of young people in Science and Technology, among other things. The current study used data generated through the application of Barômetro Brasil to 2,404 students in the age group 15 years in 2014; thus, these students were used as a representative sample of the Brazilian young population in this age group. Four (4) different typologies of Brazilian students were found based on the k-means clustering method: Reluctant, Enthusiast, Unselective Undecided, Selective Undecided. Furthermore, the interest of each typology was analyzed based on six different topics such as Health, Mystery, Scientific innovations, Agriculture, Science and Scientists, and Technology, which were determined based on the hierarchical clustering method. It was possible concluding that topics such as Mystery and Scientific innovations presented the greatest potential to boost students' intrinsic motivation, since they were the ones recording above average interest rates in all four typologies.

Keywords: Interest in Science; Intrinsic Motivation; Students' Typology; Barômetro Brasil.

\footnotetext{
${ }^{1}$ Universidade Federal de Santa Maria | daniel.ocampo@ufsm.br

${ }^{2}$ Universidade Federal de Santa Maria | Icaldeira@smail.ufsm.br
} 


\section{Introdução}

Uma queixa comum entre os docentes, inclusive de ciências, é a desmotivação dos estudantes, muitas vezes atribuída ao descompasso entre a velocidade da escola e aquela do mundo digital. Este desinteresse dos alunos, pelo conteúdo escolar, é problemático, pois como constatam Pozo e Crespo (2009, p. 40): "Os alunos não estão interessados na ciência, não querem se esforçar nem estudar e, por conseguinte, dado que aprender ciência é um trabalho intelectual complexo e exigente, fracasam".

A questão da motivação parece ser um fator fundamental para o processo de ensinoaprendizagem e que, por vezes, passa despercebido nas discussões sobre o tema. A literatura aponta duas orientações motivacionais para a aprendizagem escolar, a extrínseca e a intrínseca (Deci et al, 2001; Martinelli \& Bartholomeu, 2007; Pozo \& Crespo, 2009). A motivação extrínseca diz respeito a execussão de uma tarefa em resposta de algo externo, ou seja, o aluno se motiva em virtude de uma recompensa (nota, elogio, reconhecimento) ou para evitar punições (reprovação, castigo, humilhação). Desta forma, o estudante se motiva pois objetiva atender ao comando ou à pressão externa, de outras pessoas (Martinelli \& Bartholomeu, 2007).

Já a motivação intrínseca é inata e diz respeito a autodeterminação (Deci, et al, 2001). Quando movido pela motivação intrínseca, o estudante busca desafios e novidades, sendo a própria participação na tarefa a recompensa principal, logo, não são necessários prêmios ou pressão externa (Martinelli \& Bartholomeu, 2007). É o aprender 'por gosto'.

Mesmo que a motivação extrínseca seja recorrente, na sala de aula, observamos, na literatura, que o incentivo a este tipo de motivação pode interferir, negativamente, na motivação intrínseca (Deci et al, 2001). Além disso, um sistema de motivação extrínseca depende da manutenção dos prêmios e castigos pois, quando são retirados ou quando perdem sentido, perde-se junto os motivos para aprender. Pozo e Crespo (2009) ressaltam, que esse tipo de motivação pode gerar resultados indesejáveis, como a sensação de irrelevância do conteúdo estudado pelo aluno e o desgosto progressivo pela matéria.

São poucos os instrumentos e dados empíricos disponíveis na literatura para compreender quais são os gatilhos de motivação intrínseca (Martinelli \& Bartholomeu, 2007). Neste sentido, usamos a hipótese de que os interesses dos estudantes, pela ciência e tecnologia, tenham potencial para fomentar a motivação intrínseca nos alunos. Desta forma, ao se estudar os interesses dos jovens, teriamos, em mãos, uma ferramenta para fomentar sua motivação intrínseca.

Soma-se a isso, o distanciamento existente entre o interesse dos estudantes e o que está presente no currículo de ciências (Amestoy, 2015; Swirski \& Baram-Tsabari, 2014; Hagay, 2015). O interesse dos jovens é pouco ou nada explorado para a construção do currículo, afinal, como aponta Ravitch (2011, p.251) "[...] os reformadores do nosso tempo continuam a procurar por atalhos e respostas rápidas".

Nesta pesquisa, objetivamos elaborar tipologias para os estudantes brasileiros, no que diz respeito aos seus interesses pelas ciências e tecnologia. Assim, acreditamos que seja possível gerar dados para fomentar um ensino apoiado na motivação intrínseca dos estudantes. E, ainda, a partir do que o estudante se interessa para gerar novos interesses, em um ciclo virtuoso de envolvimento com a aprendizagem e com o conhecimento. 


\section{Histórico das pesquisas sobre o interesse em ciências}

As pesquisas a respeito do interesse dos jovens frente às ciências e tecnologia não são algo novo: em 1984, o German Institute for Science Edutacion promoveu a primeira International Conference on Interest Research, cujo o foco foi o interesse dos estudantes acerca das ciências e tecnologia (Schreiner, 2006). O encontro foi tão relevante que na mesma ocasião já foi decidido o tema da próxima conferência, que seria a relação entre interesse e gênero, uma preocupação recorrente nas pesquisas, até os dias atuais.

Em 1996, a partir de uma rede de parcerias internacionais formada em eventos como o International Organization for Science and Technology Education Symposium (IOSTE) surge o projeto Science and Scientists (SAS). Esse estudo contou com a participação de 30 pesquisadores, de 21 países. Tal projeto originou um questionário que, entre 1996 e 1999, obteve a resposta de 9350 crianças, na faixa dos 13 anos, sobre seus interesses, experiências e percepções sobre as ciências e a respeito do que aprendem na área (Sjøberg, 2000).

Em 2001, começa a ser discutido, institucionalemente, um novo projeto, sucessor do SAS, o Project ROSE (Santos-Gouw, 2013). Esse estudo foi composto por um questionário contendo 12 seções como As minhas opiniões sobre a ciência e tecnologia, Minhas experiências fora da escola e O que quero aprender. O ROSE contou com a participação de mais de 40 países, entre eles o Brasil, os quais tiveram suas aplicações documentadas nas teses de doutoramento de Tolentino-Neto (2008) e Santos-Gouw (2013).

Algumas particularidades sobre o ROSE foram observadas por pesquisadores ao redor do mundo, como a grande quantidade de questões e a falta de itens que levassem em conta o texto regional onde foi aplicado. Dessa forma, surge o projeto Barômetro Brasil. 0 Barômetro é um questionário composto por 96 questões, na escala de Likert de 4 pontos, variando de 1- Desinteressado a 4- Muito Interessado, as quais buscam respostas para diferentes aspectos do interesse e do posicionamento dos estudantes frente às ciências e tecnologia.

\section{Metodologia}

Neste trabalho, analisamos as respostas dos alunos referente à seção " $A$ " do questionário Barômetro Brasil, aplicado em 2014, a estudantes da faixa dos 15 anos e com representatividade nacional. Esta seção contempla questões referentes ao nível de interesse em aprender determinados temas. Analisamos a resposta de 2404 alunos, dos quais, 7 foram excluídos da amostra por pairwise.

Nosso objetivo é elaborar tipologias para os estudantes brasileiros, o qual pode ser atendido pela congruência de duas etapas metodológicos distintas. Em ambos os caminhos, optamos pela utilização da análise multivariada de dados, mais especificamente a análise de cluster.

A primeira etapa é da análise da forma como as questões são agrupadas, de acordo com as respostas dos jovens. Considerando, que nessa etapa, nossas variáveis seriam as 24 questões - uma amostra de poucos itens a serem agrupados -, encontramos na literatura (Hair et. al., 2009; Corrar, et. al., 2012, Ocampo, et. al., 2018) a indicação do método hierárquico como o mais adequado, utilizando o algoritmo de aglomeração do vizinho mais próximo, com a medida de distância euclidiana quadrada. 
Já a segunda etapa de análise, refere-se sobre como podemos agrupar os alunos de acordo com suas respostas. Nessa etapa, a metodologia sugerida (Hair et al., 2009; Corrar, et al., 2012, Ocampo e Tolentino-Neto, 2019) é a de k-means, método mais adequado, em virtude da quantidade massiva de dados que temos (2397 alunos). A partir de simulações e do conhecimento da amostra, definimos que deveriamos trabalhar com um total de 4 clusters no método k-means.

Destacamos, ainda, que a união dessas duas etapas é o que gera, em nossa concepção, a tipologia dos estudantes. Nos próximos itens deste texto apresentamos os resultados e discussão de cada uma dessas etapas, separadamente, logo após, apresentamos a união de ambas.

\section{Agrupamento de questões}

As 24 questões do Barômetro Brasil que analisamos podem ser agrupadas, empiricamente, por temas. Por exemplo, o item A2 "O câncer, o que sabemos e como podemos tratá-lo", pode ser classificado como uma questão que trata do tema saúde. Desta forma, acreditamos que os estudantes responderiam de acordo com as áreas de sua preferências. Para buscarmos um embasamento estatístico para esta hipótese utilizamos a análise de cluster.

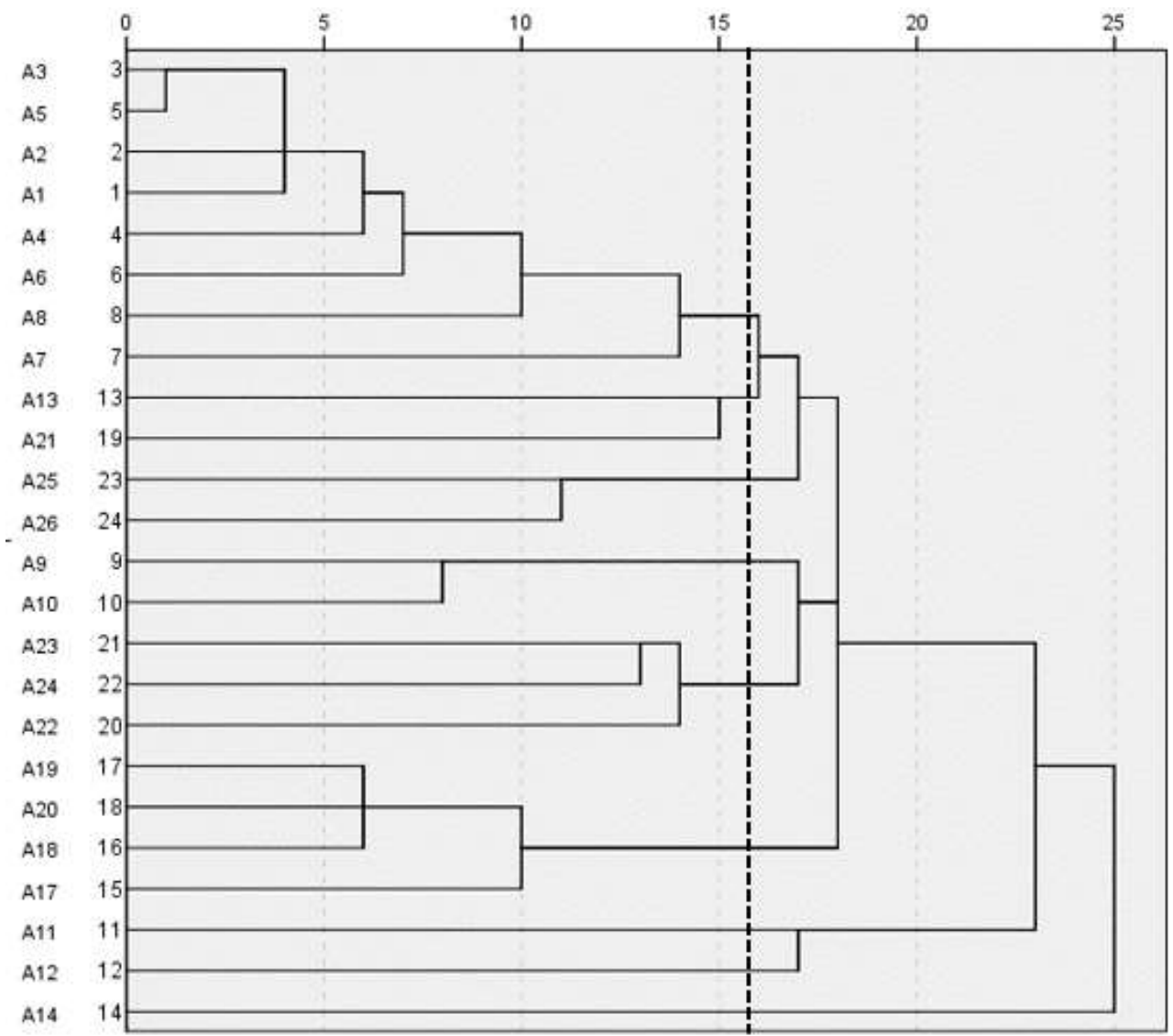

Nota: O eixo vertical representa o número de interessões e o horizontal os itens. A linha pontilhada representa os agrupamenos após 15 interações, que escolhemos analisar.

Figura 1. Dendograma dos agrupamentos das questões pelo método hierárquico do vizinho mais próximo. Fonte: Próprios autores 
Aplicamos o método hierárquico do vizinho mais próximo, o qual gerou o dendograma a seguir (Figura 1). Optamos por destacar os clusters gerados após 15 interações (Destacada por uma linha pontilhada no gráfico), em virtude da boa hetereogenidade gerada em cada cluster.

As questões A11, A12 e A14 não se agruparam a nenhuma outra questão, após 15 interações, sendo assim, optamos por desconsiderá-las para nossa discussão. Assim, a análise de cluster gerou seis agrupamentos, que classificamos de acordo com as temáticas (Tabela 1). Esses agrupamentos ficaram muito próximos dos criados por outros autores que trabalharam tanto com o Barômetro quanto com o ROSE (Schreiner, 2006; Tolentino-Neto, 2008; Chang et. al, 2009; Santos-Gouw, 2013; Pinafo, 2016). Desta forma, nomeamos os agrupamentos levando em conta as legendas utilizadas por estes autores, em suas respectivas pesquisas.

Tabela 1. Classificação do agrupamento das questões

\begin{tabular}{cc}
\hline Agrupamento & Questões \\
\hline$a-$ Saúde & $A 1, A 2, A 3, A 4, A 5, A 6, A 7, A 8$ \\
$b-$ Mistérios & $A 13, A 21$ \\
$c-$ Inovações científicas & $A 25, A 26$ \\
$d-$ Agricultura & $A 9, A 10$ \\
$e-$ Ciência e cientistas & A22, A23, A24 \\
$f-$ Tecnologia & $A 17, A 18, A 19, A 20$ \\
\hline
\end{tabular}

Fonte: Elaborado pelos autores, adaptado de Schreiner (2006), Tolentino-Neto (2008); Chang et al. (2009), Santos-Gouw (2013) e Pinafo (2016).

\section{Agrupamento dos estudantes}

Iniciamos esta seção reforçando que, entendemos cada estudante como um sujeito diferente, com seu contexto, concepções e experiências. Por outro lado, nos parece importante buscar algumas generalizações para o contexto da educação e das políticas públicas. Isto posto, agrupamos os estudantes respondentes do Barômetro Brasil de acordo com suas percepções frente às ciências.

Nosso primeiro desafio, nesta etapa, foi de definir quantos agrupamentos utilizariamos, uma vez que o método de k-means para análise de clusters, exige a determinação a priori da quantidade de agrupamentos. Recorrendo à literatura, encontramos que Schreiner (2006) - utilizando o instrumento inspiração para o Barômetro - optou pela distinção de cinco agrupamentos para os estudantes noruegueses. Já Ogawa e Shimode (2004) elaboraram três tipologias para os jovens japoneses.

Após algumas simulações e estudo dos dados, percebemos que dois agrupamentos sempre surgiam, em um deles estão os estudantes com maior interesse médio por ciências e tecnologia e em outro os jovens com menor interesse por essas. Qualquer número maior que dois sempre gera agrupamentos intermediários, que se diferenciam pela discrepância em um ou mais itens, ou seja, alto ou baixo interesse em itens específicos. Podemos aumentar este número de agrupamento até 2397, para que cada agrupamento represente 
um estudante. Em função da heterogeneidade obtida, concluímos que quatro agrupamentos seriam suficientes para segregação e definição desta amostra.

Tabela 2. Distribuição dos jovens por agrupamento

\begin{tabular}{cccc}
\hline Cluster & Frequência & Percentual (\%) & Percentual Valido (\%) \\
\hline 1 & 530 & 22,0 & 22,1 \\
2 & 574 & 23,9 & 23,9 \\
3 & 678 & 28,2 & 28,3 \\
4 & 615 & 25,6 & 25,7 \\
\hline Total & 2397 & 99,7 & 100,0 \\
\hline
\end{tabular}

Fonte: Próprios autores

O montante de jovens, em cada agrupamento, não difere tanto, sendo uma variação de 6,2\% entre os clusters 1, com menor quantidade de estudantes, e o cluster 3, com a maior quantidade de jovens (Tabela 2). Outros autores encontraram clusters com uma distribuição mais desigual, Schreiner (2006) encontrou uma variação de, aproximadamente, $13 \%$ entre os clusters com mais e menos jovens noruegueses.

Optamos por ordenar os agrupamentos de acordo com o valor médio para as respostas: o primeiro agrupamento é composto por estudantes com menor interesse pelos temas de ciências e tecnologia, enquanto o quarto agrupamento agrega jovens com maior interesse pelos temas (Figura 2).

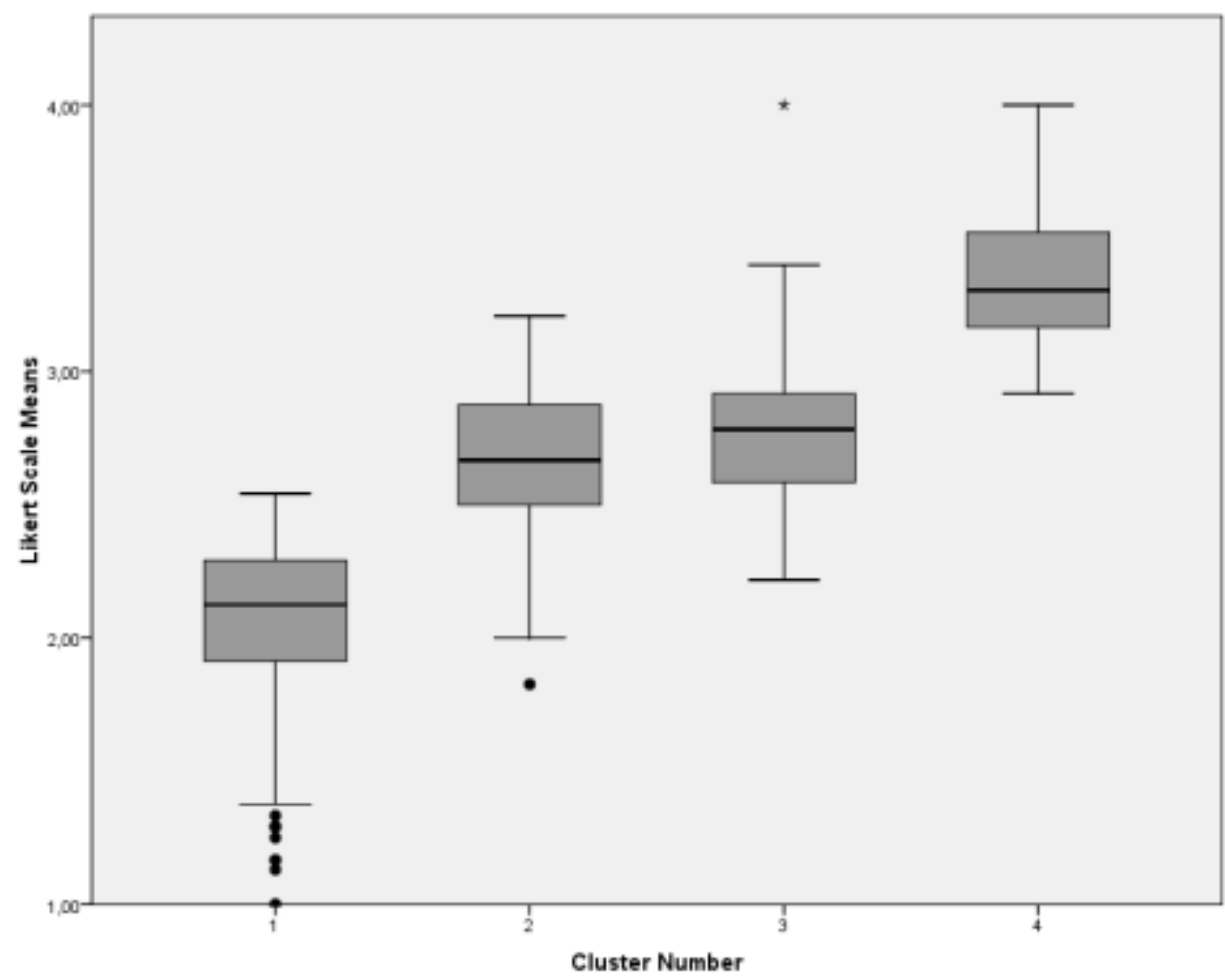

Fonte: Gráfico elaborado pelos próprios autores a partir dos resultados de PINAFO (2016). Nota: Sendo esta uma escala de Likert de quatro pontos, o mínimo possível é 1 e o máximo 4.

Figura 2. Média e quartis dos interesses por agrupamento 
É possivel observar que são poucos os outliers, que se concentram no agrupamento 1 dentre eles um jovem que apontou interesse mínimo (média 1). Por outro lado, encontramos um outlier, no agrupamento 3, que apontou muito interesse.

Para nomear esses agrupamentos, nos debruçamos, novamente, sobre os trabalhos de Ogawa e Shimode (2004) e Schreiner (2006), pesquisas que, também, criaram tipologias de estudantes japoneses e noruegueses, respectivamente. Ogawa e Shimode (2004) criaram a tipologia de estudantes japoneses utilizando dois itens do ROSE, O F2 "interesse ciência escolar" e o item F5 "gosto mais de ciências do que outras disciplinas" (Ogawa \& Shimode, 2004). Após análisar os dados, os pesquisadores chegaram a três tipologias de estudantes, um grupo dos estudantes que apresentam desinteresse pela ciência escolar, batizado de Poor Priority, e dois grupos de estudantes interessados pela ciência escolar, os Specific Priority, os quais declaram a disciplina de ciências como uma de suas preferidas, e os Other Priority, que preferem outras disciplinas à ciências.

Já os estudantes noruegueses foram divididos em 5 agrupamentos, que Schreiner (2006) distingue por três características. A primeira é a distinção por interesse, que gera três tipologias, os Reluctant, Undecided e Enthusiast. A outra distinção feita pela autora é de acordo com a variância nas respostas dos estudantes, o que os distingue como Unselective e Selective. Por fim, a pesquisadora difere os estudantes pelo o que ela chama de "Virtually sex-specific" (Schreiner, 2006, p.128). Ao fim, os jovens noruegueses puderam ser agrupados em Unselective Reluctant, Unselective Undecided, Unselective Enthusiast, Selective Boy and Selective Gril.

Considerando essas duas pesquisas, chegamos ao primeiro aspecto que distingue as tipologias dos estudantes, seus interesses pelas ciências. Optamos por utilizar os termos utilizados por Schreiner (2006), sendo assim, classificamos os estudanes do cluster 1 como Relutantes, dos cluster 2 e 3 como Indecisos, e do cluster 4 como Entusiastas.

Entretanto, defendemos que, apenas o interesse médio não é suficiente para caracterizarmos a tipologias dos estudantes. Por isso, buscamos criar uma segunda dimensão para a elaboração dessas tipologias, lançando um olhar sobre as áreas de interesse que mais representam os estudantes pertencentes a estes clusters.

\section{Tipologia dos estudantes}

Para caracterizar cada uma das tipologias dos estudantes, optamos por observar como os membros de cada um dos quatro clusters se posicionam frente aos temas apresentados na Tabela 1. Desta forma, elucidaremos as tipologias não apenas pelo interesse em ciências, mas, também, por qual tema diferencia estes clusters.

Inicialmente, nossa preocupação era distinguir as aréas de interesse de cada cluster. Entretanto, sendo os valores de interesses médios distintos, teriamos dificuldade em visualizar, eficientemente, essas diferenças. Por esse motivo, optamos por ponderar os interesses pelas áreas, através do interesse médio de cada cluster. Assim, chamaremos este valor de $\bar{u}_{\mathrm{p}}$ e o calcularemos dividindo o interesse dos jovens de cada cluster por uma determinada área pela média do interesse deste cluster. Logo, poderemos sobrepor e comparar essas grandezas, pois cada $\bar{u}_{p}>1$ representa interesse acima da média e $\bar{u}_{p}<1$ significam interesse abaixo da média. Estes resultados estão apresentados no figura 3. 


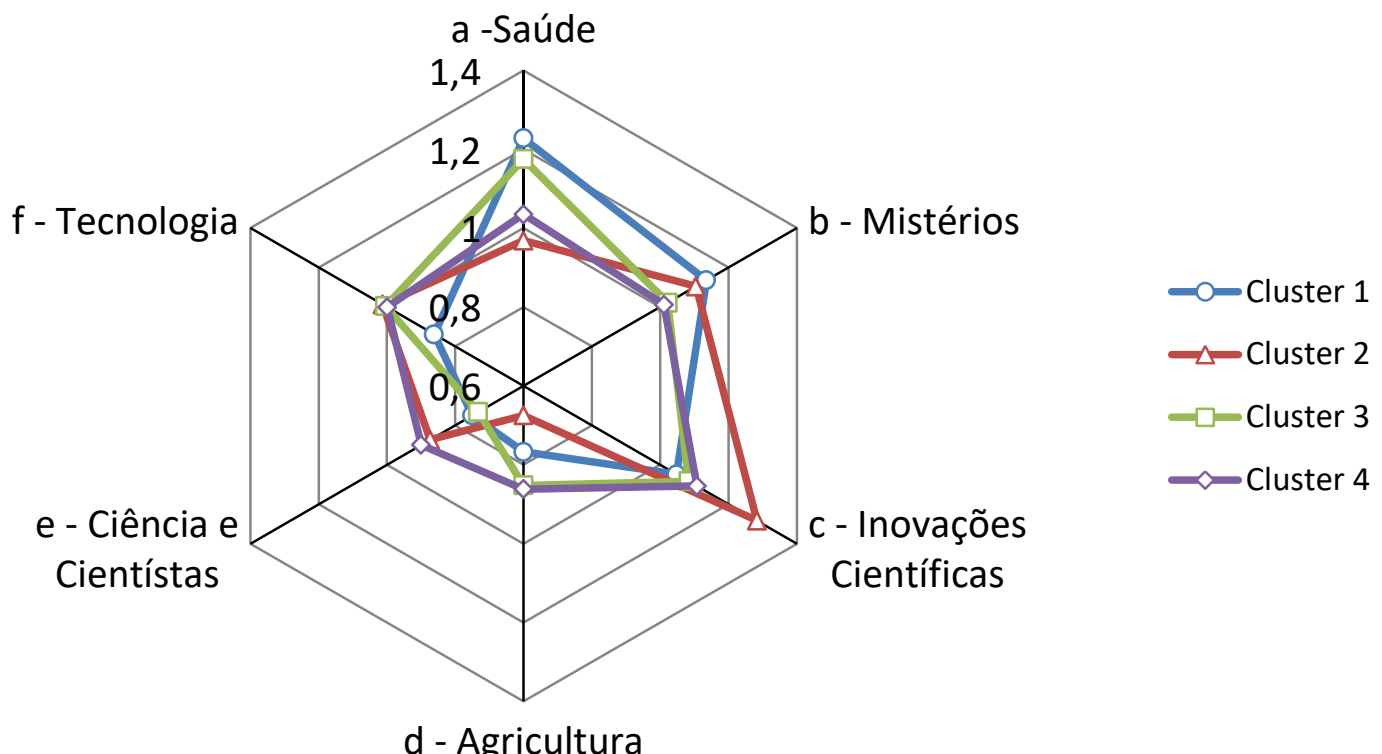

Nota: $O$ centro do radar representa $\bar{u}_{p}=0,6$ enquanto no extremo $\bar{u}_{p}=1,4$. Cada $\bar{u}_{p}>1$ representa alto interesse dos estudantes deste cluster pelo tema.

Figura 3. Comparação das médias ponderadas de interesse pelas áreas. Fonte: Próprios autores.

O dado que mais se destaca na figura 3 é o alto interesse dos estudantes, que compõem o cluster 2 pelas Inovações científicas. Além disso, é notável que os estudantes dos clusters 2 e 4 têm opiniões similares sobre as áreas Saúde, Ciência e cientistas e Tecnologia. Além disso, o cluster 2 se destaca por conter os estudantes com menor interesse pela área de Agricultura.

Um tema que aparece com interesse considerável em todos os clusters é o de Saúde, sendo que apenas o cluster 2 apresenta $\bar{u}_{p}<1$, mesmo assim muito próximo de 1 . Esse fato corrobora com resutados encontrados em outras pesquisas, como a de Santos-Gouw (2013). Entretanto, há um maior interesse dos jovens que compõem os clusters 1 e 3, em referência aos clusters 2 e 4 (Figura 3).

Para ilustrar, imaginemos uma realidade, onde a maioria dos jovens pertencesse aos clusters 1 e/ou 3, sendo assim, a utilização do tema Saúde apresentaria grande potencial, pois sensibilizaria a motivação intrínseca dos estudantes. Da mesma forma, se a realidade em questão não contar com estudantes no cluster 1, é interessante considerar a utilização do tema Tecnologia, uma vez que esse tem alto interesse dos estudantes participantes dos demais clusters. Aplicar o questionário e caracterizar/mapear a turma é uma ótima forma de o professor 'personalizar' seus planejamentos, trazendo exemplos e temáticas de interesses dos seus estudantes.

Por outro lado, ressaltamos que não é o caso de deixar de ensinar aquilo que não interessa aos estudantes. Seria negligência ou irresponsabilidade do professor deixar de abordar - por exemplo - fotossíntese, caso os alunos não exepressem interesse em botânica ou em questões ambientais. O caminho nos parece diferente e concordamos com Pozo e Crespo (2009, p.43) quando defendem que: "[...] Seria questão de partir dos interesses e preferências dos alunos para gerar outros novos". 
Desta forma, fica evidente que a identificação de cada cluster é importante, por este motivo criamos tipologias, as quais nomeiam e caracterizam cada um destes grupos. Para tanto, consideramos dois aspectos, a já citada nomeclatura por interesse médio (Relutantes, Indecisos e Entusiastas) e a área de maior interesse de cada agrupamento (Figura 4), principalmente para distinguir os clusters 2 e 3, ambos classificados como Indecisos. Ressaltamos, que na figure 4 o centro do radar representa o valor 1 (Desinteressado) e o extremo tem valor 4 (Muito Interessado).

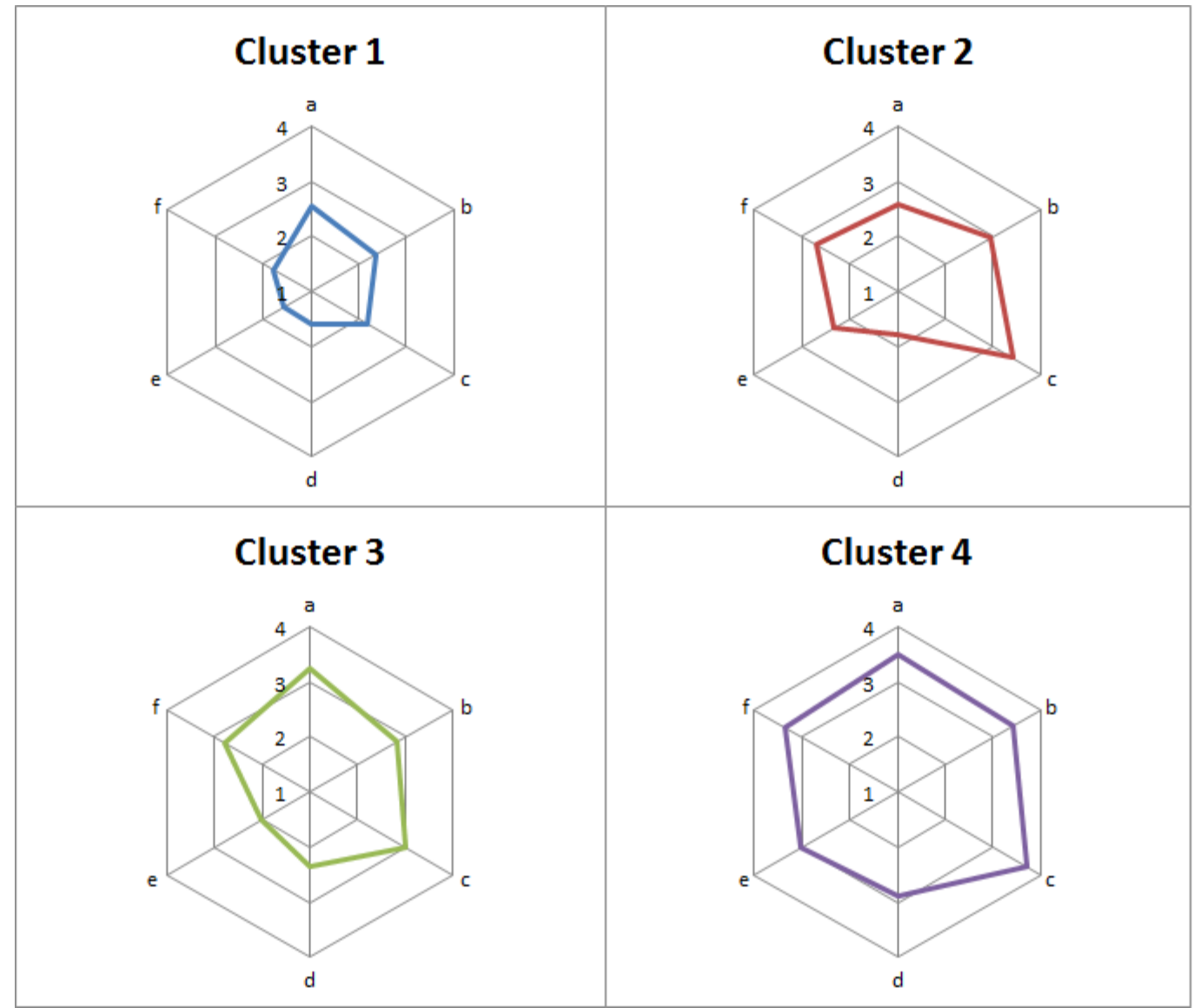

Nota: a - Saúde; b - Mistério; c - Inovações científicas; d - Agricultura; e - Ciência e cientístas; f Tecnologia

Figura 4. Tipologias de acordo com as áreas. Fonte: Próprios autores

A primeira tipologia diz respeito aos estudantes que compõem o cluster 1. Mesmo que esses jovens tenham pouco fascínio pela ciência e tecnologia, o tema saúde os atrai. Os dados apontam que a saúde desperta um interesse, aproximadamente, de 25\% maior que a média. Já o que desinteressa a esses jovens são os tópicos referentes à Agricultura, Ciência e cientistas e Tecnologia. Desta forma, nomeamos esta tipologia de Relutantes, seguindo o modelo de Schreiner (2006). Os jovens Relutantes correspondem a, aproximadamente, 22\% dos adolescentes brasileiros, da faixa dos 15 anos.

Do outro lado do espectro, temos os jovens muito interessados pelas ciências. Esses etudantes compõem o cluster 4, que representa, aproximadamente, 26\% da população brasileira, na mesma faixa etária. Esta tipologia foi nomeada Entusiastas, pois, exceto pelo tema agricultura, todos os outros apresentam interesse médio, acima dos 3 pontos, ou seja, os jovens dessa tipologia estão entre interessados e muito interessados, por quase todos os 
temas da ciência. Além do baixo interesse pelo tema agricultura, destacamos o alto interesse dos Entusiastas pelas Inovações Científicas, com uma média de $(3,72)$, muito próxima do ponto máximo. Destacamos, que é característico de jovens de países com baixo Índice de Desenvolvimento Humano (IDH), o que é a realidade de uma grande parcela dos brasileiros, apresentar uma atitude otimista e favorável frente às ciências e tecnologia (Anderson, 2006) e, por este motivo, não surpreende que uma parcela significativa dos jovens seja da tipologia Entusiastas.

As duas tipologias supracitadas englobam grande parte dos jovens brasileiros. Todavia, aproximadamente, 52\% dos estudantes fazem parte dos clusters 2 e 3, com interesse próximo da média da escala de Lickert de 4 pontos $(2,5)$. Estes dois clusters representam o que Schreiner (2006) chama de Indecisos. Desta forma, buscamos diferenciar as duas tipologias, de acordo com as particularidades de seus interesses.

Ao analisarmos as respostas dos estudantes do cluster 2, observamos que esses apresentam maior discrepância entre as respostas para cada área, quando comparados aos jovens do cluster 3, como é possivel observar na figura 1. Observamos uma variância de 0,301 entre as áreas no cluster 2, contra 0,197 do cluster 3. Desta forma, optamos por batizar o cluster 2 como Indecisos Não Seletivos e os jovens do cluster 3 como Indecisos Seletivos, mesmo critério utilizado por Schreiner (2006).

Os Indecisos Seletivos apresentam grande interesse pelas Inovações científicas e não parecem ter interesse por temas referentes à Agricultura. Esse dado nos leva a pensar como seria a representatividade destes jovens em escolas urbanas e rurais, ou mesmo em regiões de alta ou baixa densidade populacional? Com nossa amostra ainda não somos capazes de reponder esse questionamento. Da mesma forma, cidades de interior podem ter maior interesse por agricultar e pela utilização, inclusive, de recursos oriundos da produção agrícola, como por exemplo fitoterápicos e plantas medicinais, como apontado na pesquisa de Araújo e Lima (2019)

Já os jovens Indecisos Não Seletivos apresentam maior interesse pela área da Saúde e menor pela Ciência e cientistas. Algumas pesquisas anteriores apresentaram maior interesse das meninas pela área da saúde, não só no contexto brasileiro (Pinafo, 2016) mas, também, ao redor do mundo, como por exemplo na Suécia, Finlândia, Espanha e Gana (Jidesjö, et. al, 2009; Lavonen et al., 2008; Vázquez \& Manassero, 2009; Anderson, 2006). Sendo assim, optamos por verificar se havia distinção de sexo nesta tipologia: 60\% dos jovens que compõem este cluster são meninas, enquanto a tipologia Indecisos Seletivos conta com, aproximadamente, $44 \%$ de jovens meninas. Esta variação não é tão significativa como a encontrada nas pesquisas supracitadas, por este motivo, não nos debruçamos sobre esta variável quando discutimos estes dados.

Nenhuma das tipologias encontradas em nosso estudo apresentam tanta discrepância em relação ao sexo, quanto as encontradas na pesquisa de Schreiner (2006), onde, em um agrupamento, haviam 97\% de meninos e em outro 94\% de meninas. Por esta razão, não acreditamos que seja conveniente distinguir as tipologias, no Brasil, por Sex-Variable, como feito pela pesquisadora.

Assim, as tipologias que econtramos para caracterizar os jovens brasileiros são os Relutantes, Entusiastas, Indecisos Não Seletivos e Indecisos Seletivos. Evidente que essa não é uma norma, visto que: generalizações no campo do ensino, principalmente em um país tão heretogêneo quanto o Brasil, são perigosas. Por outro lado, estas tipologias podem servir 
como fio condutor, para pensarmos estratégias que levem em conta a motivação intrínseca dos estudantes, a fim de fomentar o ensino de ciências no âmbito brasileiro.

\section{Considerações finais}

O presente estudo buscou elaborar tipologias que representassem os jovens brasileiros, quanto ao interesse por ciência e tenologia. Dentro dos aspectos que pudemos quantificar, por meio do Barômetro Brasil, foi possivel segregá-los em quatro grupos: Relutantes, Indecisos Não Seletivos, Indecisos Seletivos e Entusiastas.

Compreender a existência e a característica dessas tipologias é profícuo para fomentar o ensino que pondere as motivações intrínsecas dos estudantes, para o aprendizado das ciências. Desta maneira, é possivel partir dos interesses dos estudantes para ampliar seus reportórios de conhecimentos sobre ciências.

Concluímos, neste estudo, que cada uma dessas quatro tipologias possuem interesses próprios. Os Relutantes apresentam maior interesse por Saúde, Mistérios e Inovações Científicas, similar aos jovens que compõem as tipologias Entusiastas e Indecisos Não Seletivos, os quais manifestam interesse por Inovações Científicas e Saúde, já os adolescentes da tipologia Indecisos Seletivos apresentam um elevado interesse por Inovações Científicas.

Além dos interesses individuais das tipologias, é importante observar quais são as áreas de que dispertam o interesse dos jovens de todas as tipologias. Observamos, que os itens relacionados aos Mistérios e Inovações Científicas foram os que apresentaram interesse acima da média, em todas as tipologias. Assim, reiteramos o potencial dessas duas áreas para o fomento da motivação intrínseca dos estudantes pelos temas da Ciência, uma vez que as quatro tipologias comungam desse interesse.

As tipologias e suas respectivas análises tem grande potencial para desenvolver a motivação intrínseca dos estudantes. Ressaltamos, que não é o caso de não ensinar aquilo que o aluno não tem interesse, mas, sim, utilizar os interesses para fomentar o interesse dos estudantes e abordar os diferentes assuntos, os quais precisam ser abordados nas aulas de ciências e matemática.

\section{Referências}

AMESTOY, M, B. Articulação entre os interesses dos alunos e livros didáticos: A voz do estudante na construção curricular de ciências. 2015. 82 f. Dissertação (Mestrado) - Centro de Ciências Naturais e Exatas, Universidade Federal de Santa Maria, Santa Maria. 2015.

ANDERSON, I. K. The Relevance of Science Education: As seen by Pupils in Ghanaian Junior Secondary Schools. 2006. 376f. Tese (Doutorado) - Department of Mathematics and Science Education, University of the Western Cape, Western Cape. 2006. Disponível em http://etd.uwc.ac.za/xmlui/handle/11394/1814/ acessado em 04 de maio de 2020.

ARAÚJO, Maurício dos Santos; LIMA, Michelle Mara de Oliveira. O uso de plantas medicinais para fins terapêuticos: os conhecimentos etnobotânicos de alunos de escolas pública e privada em Floriano, Piauí, Brasil. Amazônia: Revista de Educação em Ciências e Matemáticas, [S.I.], v. 15, n. 33, p. 235-250, jun. 2019. 
CHANG, S., YEUNG, Y., CHENG, M. H. Ninth Graders' Learning Interests, Life Experiences and Attitudes Towards Science \& Technology. Journal of science education and technology, 18 (5), $447-457.2009$.

.CORRAR, L. J., et. al. Análise Multivariada. 1 ed. São Paulo, SP: Atlas. 2012.

DECl, E. L., KOESTNER, R., RYAN, R. M. Extrinsic Rewards and Intrinsic Motivation in Education: Reconsidered Once Again. Review of Educational Research, 71 (1), 1-27. 2001.

HAGAY, G. A. Strategy for Incorporating Students' Interests into the High-School Science Classroom. Journal of Research in Science Teaching. 52 (7), 949 - 978. 2015.

HAIR, J. F., et. al. Análise Multivariada de Dados. Tradução de Adonai Schlup Sant'Ana. 6. Ed. Porto Alegre, RS: Bookman. 2009.

JIDESJÖ, A.; OSCARSSON, M.; KARLSSON, K. G. Science for all or science for some: What Swedish students want to learn about in secondary science and technology and their opinions on science lessons. NorDiNa, 5(2), 213-229. 2009.

LAVONEN, J., BYMAN, R., UITTO, A., JUUTI, K., MEISALO, V. Students' Interest and Experiences in Physics and Chemistry related Themes: Reflections based on a ROSE-survey in Finland. Themes in Science and Technology Education 1 (1). 7-36. 2008.

MARTINELLI, S. C., BARTHOLOMEU, D. Escala de Motivação Acadêmica: Uma Medida de Motivação Extrínseca e Intrínseca. Avaliação Psicológica, 6 (1). 21-31. 2007.

OCAMPO, D. M., et al. Diferentes Perfis de Estudantes Brasileiros Frente aos Desafios Ambientais: Resultados de uma Pesquisa de Larga Escala. Educação Ambiental em Ação, v. 65, n. 7.2018.

OCAMPO, D. M., TOLEnTINO-NETO, L. C, B. Cluster Analysis for Data Processing in Educational. Acta Scientiae, v. 21, n. 4. 2019.

OGAWA, M., SHIMODE, S. Three distinctive groups among japanese students in terms of their school science preference: from preliminary analysis of japanese data of an international survey 'the relevance of science education' (ROSE). Journal of Science Education in Japan, 28 (4). 1-11. 2004.

PINAFO, J. O que os jovens têm a dizer sobre ciências e tecnologia? Opiniões, interesses e atitudes de estudantes em dois países: Brasil e Itália. 2016. 465 f. Tese (Doutorado) Faculdade de Educação, Universidade de São Paulo, São Paulo. 2016.

POZO, J. I., CRESPO, M. A. G. A aprendizagem e o ensino de ciências: do conhecimento cotidiano ao conhecimento científico. 5ed. Porto Alegre, RS: Artmed. 2009.

RAVITCH, D. Vida e morte do grande Sistema escolar Americano: Como testes padronizados e o modelo de Mercado ameaçam a educação. Trad. de Marcelo Duarte. Porto Alegre: Sulina. 2011.

SANTOS-GOUW, A. M. As opiniões, interesses e atitudes dos jovens brasileiros frente à ciência: uma avaliação em âmbito nacional. 2013. 142 f. Tese (Doutorado) - Faculdade de Educação, Universidade de São Paulo, São Paulo. 2013. 
SCHREINER, C. Exploring a ROSE-Garden: Noewegian youth's orientations towards science see an signs of late modern identities. 2006. 315 pg. Tese (Doutorado) -Faculty of Education, University of Oslo. 2013.

SJØBERG, S. Science And Scientists: The SAS-study. Acta Didactica 1/2000, v. 1, p. 75. 2000.

SWIRSKI, H., BARAM-TSABARI, A. Bring the Gap between the Science Curriculum and Students' Questions: Comparing Linear vc. Hypermedia Online Learning Environments. Interdisciplinary Journal of E-Learning and Learning Objects. 10(1), 153 - 175. 2015. Disponível em https://www.openu.ac.il/lists/mediaserver_documents/IJELLOv10p153-175Swirski0898.pdf Acessado em 04 de maio de 2020.

TOLENTINO-NETO, L. C.B. Os interesses e posturas dos alunos frente às ciências: resultados do Projeto ROSE aplicado no Brasil. 170 f. Tese (Doutorado). Faculdade de Educação. Universidade de São Paulo. São Paulo, 2008.

VÁZQUEZ, Á:; MANASSERO, M. A. La relevância de la educación científica: actitudes y valores de los estudiantes relacionados com la ciência y la tecnologia. Enseñanza de las Ciencias, 27 (1), 33-48. 2009. 